\title{
Adesão à dieta por idosos com síndrome metabólica assistidos na Estratégia Saúde da Família: frequência e associação com depressão
}

\author{
Diet adherence in elderly patients with metabolic syndrome treated \\ by the Family Health Strategy: frequency and association with depression
}

\author{
Camila Bittencourt Jacondino' ${ }^{1}$, Vera Elizabeth Closs' ${ }^{1}$, Irênio Gomes ${ }^{1}$, Carla Helena Augustin Schwanke $₫$ \\ ' Programa de Pós-graduação em Gerontologia Biomédica do Instituto de Geriatria e Gerontologia da Pontifícia Universidade Católica \\ do Rio Grande do Sul (IGG-PUCRS). Porto Alegre, RS
}

\section{RESUMO}

Objetivos: Descrever a frequência de adesão à dieta e analisar sua associação com depressão em idosos com síndrome metabólica assistidos na atenção básica de saúde.

Métodos: Estudo transversal que incluiu idosos com síndrome metabólica diagnosticada pelos critérios do National Cholesterol Education Program Adult Treatment Panel III, participantes do Estudo Epidemiológico e Clínico dos Idosos Atendidos pela Estratégia de Saúde da Família do Município de Porto Alegre (EMI-SUS). A avaliação da adesão à dieta foi realizada através de um questionário, elaborado pelos pesquisadores com base nas recomendações da I Diretriz Brasileira de Síndrome Metabólica, sobre a adesão a oito possíveis orientações dietéticas recebidas de profissionais da saúde (em relação à ingestão de "alimentos integrais", "doces", "açúcar", "frutas/verduras/legumes", "sal", "alimentos gordurosos/frituras", "alimentos processados" e "peixes"). Considerou-se aderente à dieta o indivíduo que seguiu todas as orientações dietéticas recebidas, independentemente de quantas foram (entre uma e oito possíveis orientações). O diagnóstico de depressão foi baseado no instrumento Mini International Neuropsychiatric Interview, aplicado por psiquiatras.

Resultados: Foram incluídos 109 idosos, sendo que a maioria era do sexo feminino (67,9\%). A média da idade foi 68,4 $\pm 6,6$ anos (61-90 anos). Trinta e sete idosos $(33,9 \%)$ foram considerados aderentes à dieta. A orientação dietética com maior adesão foi a de diminuir a ingestão de sal $(89,1 \%)$ e a com menor adesão foi a de consumir alimentos integrais $(59,7 \%)$. Não se observou associação da adesão à dieta com variáveis sociodemográficas, clínicas e componentes da síndrome metabólica. A frequência de adesão à orientação de evitar o consumo de açúcar e de doces foi significativamente menor entre os indivíduos com depressão ( $43,9 \%$ vs $34,9 \%$; $p=0,001)$.

Conclusões: A adesão à dieta foi observada em um terço dos idosos com síndrome metabólica atendidos na atenção básica de saúde. Nos indivíduos com diagnóstico de depressão, a adesão às orientações de evitar o consumo de doces e de açúcar foi menor.

DESCRITORES: idoso; síndrome X metabólica; comportamento alimentar; depressão; atenção primária à saúde.

\section{ABSTRACT}

Aims: To describe diet adherence and to analyze its association with depression in elderly patients with metabolic syndrome treated by the Family Health Strategy.

Methods: Cross-sectional study including elderly patients with metabolic syndrome diagnosed according to the National Cholesterol Education Program Adult Treatment Panel III criteria who participated in the Epidemiological and Clinical Study of Elderly Individuals Treated by the Family Health Strategy in Porto Alegre, Brazil. A questionnaire developed by the researchers based on the recommendations of the I Brazilian Guideline for Metabolic Syndrome was used to evaluate compliance with eight possible dietary guidelines provided by health professionals (intake of "whole foods", "sweets", "sugar", "fruits/vegetables/legumes", "salt", "fatty foods/fried foods", "processed foods" and "fish"). Diet adherence was considered when an individual followed all dietary guidelines (regardless of their number, which ranged from one to eight). The diagnosis of depression was based on the Mini International Neuropsychiatric Interview instrument, used by psychiatrists.

Results: A total of 109 elderly patients were included in the study, most of whom were female (67.9\%). The mean age was $68.4 \pm 6.6$ years (61-90 years). Thirty-seven elderly patients $(33.9 \%)$ were considered adherent to the diet. Lower salt intake was the dietary guidance with greater adherence $(89.1 \%)$ while the consumption of whole foods exhibited less adherence $(59.7 \%)$. There was no association of diet adherence with sociodemographic and clinical parameters and with metabolic syndrome components. The frequency of adherence to the recommendation against the consumption of sugar and sweets was significantly lower among individuals with depression (43.9\% vs $34.9 \%$; $\mathrm{p}=0.001)$.

Conclusions: Diet adherence occurred in one third of elderly patients with metabolic syndrome treated at the primary health care level. There was lower adherence to the recommendation against the consumption of sweets and sugar in individuals diagnosed with depression.

KEY WORDS: elderly; metabolic syndrome X; feeding behavior; depression; primary health care. 
Abreviaturas: NCEP-ATPIII, National Cholesterol Education Program Adult Treatment Panel III; SM, síndrome metabólica; EMI-SUS, Estudo Epidemiológico e Clínico dos Idosos Atendidos pela Estratégia de Saúde da Família do Município de Porto Alegre; IMC, índice de massa corporal; HDL-c, colesterol ligado à lipoproteína de alta densidade; LDL-c, colesterol ligado à lipoproteína de baixa densidade; M.I.N.I, Mini International Neuropsychiatric Interview; IGG-PUCRS, Instituto de Geriatria e Gerontologia da Pontifícia Universidade Católica do Rio Grande do Sul.

\section{INTRODUÇÃO}

A população mundial vem passando por um processo de transição demográfica, devido à diminuição da mortalidade e das taxas de natalidade. Como consequência, houve o aumento da expectativa de vida e crescimento do número de pessoas com idade igual ou acima de 60 anos. Associada a este fenômeno, encontra-se a transição epidemiológica, com o incremento de doenças crônicas não transmissíveis, tais como as cardiovasculares e metabólicas (como diabetes mellitus e obesidade), que se destacam pela elevada incidência epidemiológica e clínica, sendo responsáveis por $82 \%$ de óbitos nesse grupo etário da população. Além disso, essas doenças comumente relacionam-se a outras afecções de grande incidência nos idosos, como os transtornos neuropsiquiátricos, entre os quais a depressão [1-3].

A adiposidade abdominal e a intolerância à glicose favorecem o surgimento da síndrome metabólica (SM), que é de etiologia multifatorial e complexa, com uma prevalência em torno de $10 \%$ a $40 \%$, dependendo da população estudada e do critério diagnóstico utilizado. A SM ocorre mais frequentemente em populações caracterizadas por ingestão excessiva de nutrientes, inatividade física e em idosos, constituindo um dos maiores desafios para a saúde pública, uma vez que aumenta a mortalidade geral em 1,5 vezes e a mortalidade cardiovascular em 2,5 vezes $[4,5]$.

A dieta adequada é um dos tratamentos de primeira escolha para o manejo da SM, pois provoca a redução do peso e da gordura visceral, exercendo efeito direto no perfil lipídico, diminuição dos níveis séricos da glicemia de jejum, assim como a redução da pressão arterial. Contudo, para o êxito do tratamento, torna-se fundamental a adesão do paciente à dieta recomendada para esses casos, que inclui consumir fibras, cereais integrais, legumes, frutas e peixes, diminuir o consumo de sal e evitar o consumo de açúcar, doces, frituras e embutidos [4-6].
A Organização Mundial da Saúde, em 2003, definiu adesão ao tratamento como a extensão pela qual o comportamento de uma pessoa que toma medicamentos, segue uma dieta ou muda seu estilo de vida, corresponde às recomendações estabelecidas pelo profissional da saúde responsável pelo tratamento do paciente [7]. A adesão às propostas de modificação do estilo de vida, sobretudo no que se refere às práticas alimentares, é geralmente muito baixa [8,9]. Além disso, fatores psicológicos podem ser relevantes no comportamento alimentar, particularmente em indivíduos com diagnóstico de depressão, uma vez que a presença de algumas manifestações desse transtorno de humor, como distorções do apetite, sentimento de inutilidade, baixa autoestima, fadiga, perda de energia e de motivação, podem implicar na adesão ao tratamento $[10,11]$.

Assim, o presente estudo teve como propósito descrever a frequência de adesão à dieta e analisar sua associação com depressão, em idosos com SM assistidos na Estratégia Saúde da Família de Porto Alegre.

\section{MÉTODOS}

\section{Delineamento, população e amostra}

Trata-se de um estudo transversal, descritivo e analítico, realizado com idosos (indivíduos com idade igual ou superior a 60 anos) participantes do Estudo Epidemiológico e Clínico dos Idosos Atendidos pela Estratégia de Saúde da Família do Município de Porto Alegre (EMI-SUS). Esse estudo amplo vem sendo desenvolvido por uma equipe de pesquisadores do Instituto de Geriatria e Gerontologia da PUCRS (IGG-PUCRS), sendo que detalhes dos métodos se encontram descritos em Gomes et al. [12].

A amostra inicial do presente estudo foi composta por 110 idosos com diagnóstico de SM pelo National Cholesterol Education Program Adult Treatment Panel III (NCEP-ATPIII) [13] que foram selecionados para participar do subprojeto "Adesão ao Tratamento Medicamentoso e Não Medicamentoso em Idosos Portadores de Síndrome Metabólica Acompanhados na Estratégia Saúde da Família" [14]. Os critérios do NCEP-ATP III incluem a associação de três ou mais dos seguintes componentes: triglicerídeos elevados $(>150 \mathrm{mg} / \mathrm{dl})$; colesterol ligado a lipoproteínas de alta densidade (HDL-c) diminuído (homens <40mg/dl e mulheres $<50 \mathrm{mg} / \mathrm{dl}$ ); pressão arterial elevada (sistólica $>135 \mathrm{mmHg}$ e diastólica $>85 \mathrm{mmHg}$ ) ou uso de medicamentos anti-hipertensivos; glicemia de jejum elevada $(>110 \mathrm{mg} / \mathrm{dl})$ ou uso de hipoglicemiante 
oral/insulina; e obesidade central (circunferência da cintura, homens $>102 \mathrm{~cm}$ e mulheres $>88 \mathrm{~cm}$ ) $[4,13]$. Não foram incluídos idosos com declínio cognitivo e com alteração na comunicação (na fala e/ou na audição) que impossibilitasse a avaliação da adesão à dieta.

\section{Variáveis, instrumentos e logística do estudo}

O estudo foi realizado em três etapas. Na primeira etapa foi aplicado o Questionário de Avaliação Global do Idoso do EMI-SUS. Na segunda etapa, no período de março a dezembro de 2011, foi realizada a coleta de dados, que incluiu as variáveis detalhadas mais abaixo, e foi realizada a avaliação psiquiátrica, além do perfil bioquímico. Essa etapa compreendeu também a identificação dos indivíduos com SM pelo critério do NCEP-ATP III. Na terceira etapa, realizada no período de junho a setembro de 2012, foi feita a aplicação do instrumento da adesão à dieta.

Variáveis sociodemográficas: idade, sexo, renda e escolaridade foram obtidas do Questionário de Avaliação Global do Idoso do EMI-SUS, padronizado para esse estudo. As informações foram coletadas pelos agentes de saúde das unidades de Estratégia Saúde da Família, no domicílio dos idosos. Após, ao longo da pesquisa, estes questionários eram enviados para o Laboratório de Bioquímica, Genética Molecular e Parasitologia (LABGEMP) do Instituto de Geriatria e Gerontologia da PUCRS, onde eram escaneados e, posteriormente, os dados eram digitados em um banco específico.

Medidas antropométricas: as variáveis antropométricas foram coletadas por uma equipe de nutricionistas, no Hospital São Lucas da PUCRS. O peso e a altura foram avaliados em uma plataforma Filizola $^{\circledR}$, com os participantes vestindo um avental hospitalar, descalços, posicionados no centro da plataforma da balança com os braços estendidos ao longo do corpo e posição ereta. $\mathrm{O}$ índice de massa corporal (IMC) foi calculado a partir das medidas de peso e altura utilizando-se o índice de Quetelet $\left(\mathrm{IMC}=\right.$ peso/altura $\left.{ }^{2}\right)$ sendo os pontos de corte para magreza e sobrepeso, respectivamente, IMC abaixo de $22 \mathrm{~kg} / \mathrm{m}^{2}$ e acima de $27 \mathrm{~kg} / \mathrm{m}^{2}$ [15]. A circunferência abdominal foi aferida com fita métrica inelástica no ponto médio entre o rebordo costal e a crista ilíaca com o indivíduo em pé, obedecidos os pontos de corte para homens $\geq 102 \mathrm{~cm}$ e para mulheres $\geq 88 \mathrm{~cm}$ [4].

Variáveis bioquímicas: foram analisados os níveis séricos de colesterol total, $H D L-c$, colesterol ligado a lipoproteínas de baixa densidade (LDL-c), triglicerídeos e glicose. As coletas de sangue venoso foram realizadas nas unidades básicas de saúde com os voluntários posicionados de acordo com as recomendações da Sociedade Brasileira de Patologia Clínica e Medicina Clínica Laboratorial [16]. Para tanto, os idosos foram orientados a manter 12 horas de jejum. As análises bioquímicas foram realizadas por pesquisadores do Laboratório de Bioquímica, Genética Molecular e Parasitologia do IGG-PUCRS.

Pressão arterial: os níveis pressóricos foram obtidos por acadêmicos de medicina, com um esfigmomanômetro aneroide devidamente calibrado e estetoscópio, com o paciente sentado em uma cadeira com as costas apoiadas, sendo conferidas as informações referentes à não realização de esforço físico, fumo, ou ingestão de cafeína, durante uma hora antes da aferição da pressão [17]. Essas medidas foram realizadas no Hospital São Lucas da PUCRS.

Depressão: a depressão foi avaliada por meio do instrumento Mini International Neuropsychiatric Interview (M.I.N.I.), uma entrevista padronizada que explora os principais transtornos psiquiátricos do eixo I do Manual de Diagnóstico e Estatística dos Transtornos Mentais - Quarta Edição (DSM-IV) [18] e da Classificação Estatística Internacional de Doenças e Problemas Relacionados à Saúde (CID-10). A entrevista foi realizada por psiquiatras, no Hospital São Lucas da PUCRS.

Avaliação da adesão à dieta: para identificação da adesão à dieta foi aplicado o Instrumento de Avaliação de Adesão à Dieta (Quadro 1) elaborado pelos pesquisadores com base na I Diretriz Brasileira de Diagnóstico e Tratamento da Síndrome Metabólica [4]. O instrumento contém perguntas com opção de resposta dicotômica ( $\operatorname{sim} /$ não) que questionam, primeiro, se o indivíduo recebeu uma determinada orientação dietética de um profissional da área da saúde e, logo após, nos casos positivos, se aderiu a essa orientação. As possíveis orientações incluídas no questionário referem-se à ingestão de oito grupos de alimentos: "alimentos integrais" (aveia, massa, pão e arroz integral); "frutas/legumes/verduras"; "açúcar"; "doces" (guloseimas, biscoitos doces recheados, refrigerantes); "alimentos gordurosos e frituras" (carne vermelha, gorduras visíveis, pele de frango, leite integral, queijo amarelo, frituras); "sal"; "alimentos processados" (embutidos, conservas, enlatados, defumados e salgados de pacote) e "peixes" (incluindo salmão, sardinha e outros peixes).

Foram considerados aderentes à dieta os idosos que seguiram todas as orientações dietéticas que referiram ter recebido de profissionais da saúde (entre uma a oito das possíveis orientações). A pergunta 
Quadro 1. Instrumento de avaliação de adesão à dieta.

\begin{tabular}{|c|c|c|}
\hline INSTRUMENTO DE AVALIAÇÃO DE ADESÃO À DIETA & \multirow[t]{2}{*}{$\operatorname{sim}$} & \multirow[t]{2}{*}{ não } \\
\hline $\begin{array}{l}\text { Q 1. O(A) senhor(a) já foi orientado(a) a seguir algum tipo de dieta? } \\
\text { Caso negativo, pule para } 4\end{array}$ & & \\
\hline $\begin{array}{l}\text { Q 2. Por quem o senhor(a) foi orientado(a) a modificar a dieta? } \\
\text { (1) médico ( } 2 \text { ) nutricionista ( } 3 \text { ) endocrinologista ( } 4 \text { ) outro }\end{array}$ & & \\
\hline Q 3. Caso a resposta seja outro, quem lhe orientou: & & \\
\hline $\begin{array}{l}\text { Q 4. } \mathrm{O}(\mathrm{A}) \text { senhor(a) foi orientado(a) para aumentar o consumo de alimentos rico em fibras como aveia, massa, pão e arroz integral? } \\
\text { Caso negativo, pule para questão } 6\end{array}$ & & \\
\hline Q 5. O(A) senhor(a) segue esta orientação? & & \\
\hline $\begin{array}{l}\text { Q 6. O(A) senhor(a) foi orientado(a) para aumentar o consumo de legumes, frutas e verduras? } \\
\text { Caso negativo, pule para questão } 8\end{array}$ & & \\
\hline Q 7. Caso foi orientado(a) o(a) senhor(a) segue esta orientação? & & \\
\hline $\begin{array}{l}\text { Q 8. O(A) senhor(a) foi orientado(a) para diminuir o consumo de açúcar simples? } \\
\text { Caso negativo, pule para questão } 10\end{array}$ & & \\
\hline Q 9. $O(A)$ senhor(a) segue esta orientação? & & \\
\hline $\begin{array}{l}\text { Q 10. O(A) senhor(a) foi orientado(a) a evitar guloseimas, biscoitos doces recheados e refrigerantes? } \\
\text { Caso negativo, pule para a questão } 12\end{array}$ & & \\
\hline Q 11. $O(A)$ senhor(a) segue esta orientação? & & \\
\hline $\begin{array}{l}\text { Q 12. O(A) senhor(a) foi orientado(a) a evitar o consumo de carne vermelha, gorduras visíveis, pele de frango, leite integral, queijo amarelo e frituras? } \\
\text { Caso negativo, pule para } \mathbf{1 4}\end{array}$ & & \\
\hline Q 13. $O(A)$ senhor(a) segue esta orientação? & & \\
\hline $\begin{array}{l}\text { Q 14. O(A) senhor(a) foi orientado(a) a diminuir o consumo de sal? } \\
\text { Caso negativo, pule a questão } 16\end{array}$ & & \\
\hline Q 15. $O(A)$ senhor(a) segue esta orientação? & & \\
\hline $\begin{array}{l}\text { Q 16. O(A) senhor(a) foi orientado(a) a evitar alimentos processados como embutidos, conservas, enlatados, defumados e salgados de pacote? } \\
\text { Caso negativo, pule a questão } \mathbf{1 8}\end{array}$ & & \\
\hline Q 17. $O(A)$ senhor(a) segue esta orientação? & & \\
\hline $\begin{array}{l}\text { Q 18. O(A) senhor(a) foi orientado(a) aumentar o consumo de alimentos ricos em ômega } 3 \text { como peixes, salmão, sardinha, cavala e arenque? } \\
\text { Caso negativo, pule para a questão } 20\end{array}$ & & \\
\hline Q 19. $O(A)$ senhor(a) segue esta orientação? & & \\
\hline $\begin{array}{l}\text { Q 20. Caso o(a) senhor(a) não tenha seguido as orientações em relação à dieta, por qual motivo não seguiu? (pode ser múltipla escolha) } \\
\text { ( ) devido a recursos financeiros } \\
\text { ( ) por não achar necessário } \\
\text { ( ) por não ter persistência } \\
\text { ( ) não sou responsável pelo preparo das refeições } \\
\text { ( ) outro motivo? Qual: }\end{array}$ & & \\
\hline
\end{tabular}

Fonte: os autores.

relacionada com os motivos para a não adesão às orientações da dieta foi de múltipla escolha, sendo a última alternativa de resposta aberta. $\mathrm{O}$ questionário de adesão foi aplicado pela primeira autora do estudo em 19 unidades de Estratégia Saúde da Família, com horário marcado com os coordenadores das unidades básicas, entre junho e setembro de 2012. Nos casos de idosos impossibilitados de se deslocarem até o local da coleta, foi realizada visita domiciliar.

\section{Análise estatística}

O banco de dados foi estruturado em planilha Microsoft Excel, e as análises realizadas através do programa SPSS Statistics 17. Foram utilizados o teste $\mathrm{t}$ de Student para variáveis contínuas e os testes qui-quadrado de Pearson ou exato de Fisher para variáveis categóricas. As análises foram realizadas considerando-se o nível de confiança de $95 \%(\alpha=5 \%)$.

\section{Aspectos éticos}

O estudo teve aprovação do Comitê de Ética em Pesquisa (CEP) da PUCRS (protocolo no 11/05629). O projeto EMI-SUS foi apreciado e aprovado pelo CEP da PUCRS (protocolo no 10/04967) e pelo CEP da Secretaria Municipal de Saúde de Porto Alegre (registro 499 e processo $\mathrm{n}$ - 001.021434.10.7). Todos os participantes assinaram um termo de consentimento livre e esclarecido. 


\section{RESULTADOS}

A amostra inicial foi composta por 110 idosos portadores de SM diagnosticada pelo critério NCEPATPIII, entretanto, um idoso foi excluído por ter alegado, posteriormente, que não havia recebido qualquer tipo de orientação nutricional, diferentemente do informado inicialmente. A amostra final, então, ficou constituída de 109 idosos, com média de idade de 68,3 $\pm 6,6$ anos no intervalo de 61 a 90 anos.

Ao analisar a adesão à dieta, 72 idosos (66,1\%) foram classificados como não aderentes e 37 idosos (33,9\%; IC 95\% 25\%-42,7\%) considerados aderentes uma vez que referiram seguir todas as orientações fornecidas pelos profissionais da saúde relativas a um ou mais dos oito grupos de alimentos.

A maior parte dos idosos estudados era do sexo feminino $(67,9 \%)$, encontrava-se na faixa etária entre 60-69 anos (63,3\%), recebia até um salário mínimo mensal (52,3\%) e tinha ensino fundamental incompleto $(75,2 \%)$. No que se refere à depressão, $35,8 \%$ dos idosos foram diagnosticados com esse transtorno. Não foi observada associação estatisticamente significativa entre adesão à dieta e variáveis sociodemográficas, bem como com depressão (Tabela 1).

Em relação às frequências dos componentes da $\mathrm{SM}, 41,3 \%$ dos idosos apresentavam, pelo menos, três critérios para diagnóstico da SM, sendo que a hipertensão arterial foi o componente mais frequente $(83,5 \%)$ seguido do aumento da circunferência da cintura $(77,1 \%)$ e glicemia alterada $(60,5 \%)$. Não foi observada associação estatisticamente significativa entre a adesão a todas as orientações fornecidas (uma a oito) e os componentes da SM (Tabela 2).

As recomendações que tiveram adesão mais frequente foram "diminuir o uso de sal" $(89,1 \%)$, "aumentar o consumo de frutas e legumes" $(86,9 \%)$, "evitar embutidos"( $83,8 \%$ ) e "evitar açúcar" $82,5 \%$ (Tabela 3).

Tabela 1. Distribuição das frequências das características sociodemográficas e depressão, segundo a adesão à dieta dos idosos portadores de síndrome metabólica assistidos na Estratégia Saúde da Família de Porto Alegre, RS (N=109).

\begin{tabular}{|c|c|c|c|c|}
\hline \multirow[b]{2}{*}{ Variáveis } & \multirow{2}{*}{$\begin{array}{c}\text { Amostra total } \\
\mathrm{n}(\%)\end{array}$} & \multicolumn{2}{|c|}{ Adesão à dieta } & \multirow[b]{2}{*}{$\mathbf{p}$} \\
\hline & & $\begin{array}{l}\text { Sim } \\
\text { n (\%) }\end{array}$ & $\begin{array}{l}\text { Não } \\
\text { n (\%) }\end{array}$ & \\
\hline \multicolumn{5}{|l|}{ Sexo } \\
\hline Masculino & $35(32,1)$ & $15(42,9)$ & $20(57,1)$ & \multirow{2}{*}{$0,129^{*}$} \\
\hline Feminino & $74(67,9)$ & $22(29,7)$ & $52(70,3)$ & \\
\hline \multicolumn{5}{|l|}{ Faixa Etária } \\
\hline $60-69$ anos & $69(63,3)$ & $26(37,7)$ & $43(62,3)$ & \multirow{3}{*}{$0,311^{+}$} \\
\hline 70-79 anos & $35(32,1)$ & $09(25,7)$ & $26(74,3)$ & \\
\hline 80 anos ou mais & $05(4,6)$ & $02(25)$ & $03(75)$ & \\
\hline \multicolumn{5}{|l|}{ Renda } \\
\hline Sem renda própria & $14(12,8)$ & $7(50)$ & $7(50)$ & \multirow{5}{*}{$0,323^{\dagger}$} \\
\hline Até 1 salário mínimo & $57(52,3)$ & $18(31,6)$ & $39(68,4)$ & \\
\hline Entre 1 e 2 salários mínimos & $29(26,6)$ & $11(37,9)$ & $18(62,1)$ & \\
\hline Entre 2 e 4 salários mínimos & $05(4,6)$ & $0(0)$ & $5(100)$ & \\
\hline Não informou renda & $04(3,7)$ & $01(25)$ & $03(75)$ & \\
\hline \multicolumn{5}{|l|}{ Escolaridade } \\
\hline Analfabeto & $09(8,3)$ & $2(22,2)$ & $07(77,8)$ & \multirow{6}{*}{$0,053^{+}$} \\
\hline Fundamental incompleto & $82(75,2)$ & $25(30,5)$ & $57(69,5)$ & \\
\hline Fundamental completo & $06(5,6)$ & $5(83,3)$ & $01(16,7)$ & \\
\hline Médio incompleto & $01(0,9)$ & $1(100)$ & $0(0)$ & \\
\hline Médio completo & $09(8,3)$ & $4(44,4)$ & $5(55,6)$ & \\
\hline Não informou escolaridade & $02(1,8)$ & $0(0)$ & 02 (100) & \\
\hline \multicolumn{5}{|l|}{ Depressão } \\
\hline Sim & $39(35,8)$ & $9(23,1)$ & $30(76,9)$ & \multirow{2}{*}{$0,056^{*}$} \\
\hline Não & $70(64,2)$ & $28(40,0)$ & $42(60,0)$ & \\
\hline
\end{tabular}

* Teste Exato de Fisher. ${ }^{+}$Teste do Qui-quadrado de Pearson. 
Tabela 2. Associação entre adesão a pelo menos uma das orientações fornecidas dos grupos alimentares e componentes da síndrome metabólica em 109 idosos assistidos na Estratégia Saúde da Família de Porto Alegre, RS.

\begin{tabular}{|c|c|c|c|c|}
\hline \multirow[b]{2}{*}{ Componentes da Síndrome Metabólica } & \multirow{2}{*}{$\begin{array}{c}\text { Amostra total } \\
(\mathbf{n}=109) \\
\mathrm{n}(\%)\end{array}$} & \multicolumn{2}{|c|}{ Adesão à dieta } & \multirow[b]{2}{*}{$\mathbf{p}$} \\
\hline & & $\begin{array}{c}\operatorname{Sim}_{(\mathbf{n}=37)} \\
\mathbf{n}(\%)\end{array}$ & $\begin{array}{c}\text { Não } \\
(\mathbf{n}=72) \\
\mathbf{n}(\%)\end{array}$ & \\
\hline \multicolumn{5}{|l|}{ Pressão arterial elevada e/ou uso de anti-hipertensivos } \\
\hline Sim & $91(83,5)$ & $31(34,1)$ & $60(65,9)$ & $0,591^{\dagger}$ \\
\hline \multicolumn{5}{|c|}{ Glicemia alterada e/ou uso de hipoglicemiante oral/insulina } \\
\hline Sim & $66(60,5)$ & $22(33,3)$ & $44(66,7)$ & $0,514^{*}$ \\
\hline \multicolumn{5}{|l|}{ Triglicerídeos aumentados } \\
\hline Sim & $56(51,4)$ & $19(33,9)$ & $37(66,1)$ & $0,578^{*}$ \\
\hline \multicolumn{5}{|l|}{ HDL diminuído } \\
\hline $\operatorname{Sim}$ & $57(52,3)$ & $16(28,1)$ & $41(71,9)$ & $0,225^{*}$ \\
\hline \multicolumn{5}{|l|}{ Obesidade central } \\
\hline $\operatorname{Sim}$ & $84(77,1)$ & $25(29,4)$ & $60(70,6)$ & $0,053^{*}$ \\
\hline \multicolumn{5}{|l|}{ Componentes da SM } \\
\hline 3 componentes & $45(41,3)$ & $17(37,8)$ & $28(62,2)$ & \\
\hline 4 componentes & $41(37,6$ & $14(34,1)$ & $27(65,9)$ & $0,628^{+}$ \\
\hline 5 componentes & $23(21,1)$ & $06(26,1)$ & $17(73,9)$ & \\
\hline
\end{tabular}

SM=Síndrome Metabólica; * Teste Exato de Fisher; † Teste Qui-quadrado de Pearson.

Tabela 3. Distribuição da frequência da adesão às orientações em relação aos itens da dieta baseada nas recomendações da I Diretriz de Síndrome Metabólica em idosos assistidos na Estratégia Saúde da Família de Porto Alegre, RS (n=109).

\begin{tabular}{|c|c|c|c|}
\hline \multirow[b]{2}{*}{ Orientações } & \multirow[b]{2}{*}{$\begin{array}{c}\text { Amostra } \\
\text { n (\%) }\end{array}$} & \multicolumn{2}{|c|}{ Adesão à orientação } \\
\hline & & $\underset{\mathrm{n}(\%)}{\operatorname{Sim}}$ & $\begin{array}{c}\text { Não } \\
\text { n (\%) }\end{array}$ \\
\hline Consumir alimentos ricos em fibras (aveia, massa, pão e arroz integral) & $77(70,0)$ & $46(59,7)$ & $31(40,3)$ \\
\hline Consumir frutas, legumes e verduras & $99(90,8)$ & $86(86,9)$ & $13(13,1)$ \\
\hline Evitar açúcar & $80(72,7)$ & $66(82,5)$ & $14(17,5)$ \\
\hline Evitar doces (guloseimas, biscoitos doces recheados e refrigerantes) & $82(74,5)$ & $63(76,8)$ & $19(23,2)$ \\
\hline Diminuir sal & $101(91,8)$ & $90(89,1)$ & $11(10,9)$ \\
\hline Evitar frituras, carne vermelha, gorduras visíveis, pele de frango, leite integral, queijo amarelo & $98(89,1)$ & $77(78,6)$ & $21(21,4)$ \\
\hline Evitar embutidos, conservas, enlatados, defumados, salgados de pacote & $68(61,8)$ & $57(83,8)$ & $11(16,2)$ \\
\hline Consumir peixes & $85(77,3)$ & $61(71,8)$ & $24(28,2)$ \\
\hline
\end{tabular}

Entre os motivos para não adesão à dieta (Tabela 4), os mais frequentemente apontados pelos idosos foram "falta de persistência" (38,9\%), e "contexto perceptível” (20,8\%). Esta última justificativa relaciona-se aos aspectos palatáveis dos alimentos, ou seja, os idosos não acharem os alimentos integrais saborosos, optando pelos alimentos de farinha branca, preferindo o açúcar ao invés do adoçante e adicionando mais sal do que o recomendado, pois salientam não sentir o alimento apetitoso na ausência ou na redução desses elementos. A ausência de persistência foi justificada ainda por outros motivos (15,3\%), entre eles a falta de recursos financeiros, por considerarem desnecessária a medida, e pelo contexto perceptível nos alimentos.
Tabela 4. Distribuição da frequência dos motivos para não seguimento da dieta entre os idosos portadores de síndrome metabólica, assistidos na Estratégia Saúde da Família de Porto Alegre, RS $(n=72)$.

\begin{tabular}{lc}
\hline \multicolumn{1}{c}{ Motivos para não adesão à dieta } & $\mathbf{n}(\%)$ \\
\hline Falta de persistência & $28(38,9)$ \\
Não achar necessário & $10(13,9)$ \\
Contexto perceptível* & $15(20,8)$ \\
Falta de recursos financeiros & $6(8,3)$ \\
Falta de persistência + contexto perceptível* & $6(8,3)$ \\
Falta de persistência + falta de recursos financeiros & $3(4,2)$ \\
Falta de persistência + não achar necessário & $2(2,8)$ \\
Não achar necessário + contexto perceptível* & $2(2,8)$ \\
\hline
\end{tabular}

* Aspectos palatáveis dos alimentos. 
Tabela 5. Associação entre depressão e adesão a cada recomendação dietética em idosos portadores de síndrome metabólica assistidos na Estratégia Saúde da Família de Porto Alegre, RS (n=109).

\begin{tabular}{|c|c|c|c|}
\hline \multirow{2}{*}{ Orientações } & \multicolumn{2}{|c|}{ Depressão } & \multirow{2}{*}{$\mathbf{p}^{*}$} \\
\hline & $\underset{\mathbf{n}(\%)}{\operatorname{Sim}}$ & $\begin{array}{l}\text { Não } \\
\text { n (\%) }\end{array}$ & \\
\hline Consumir fibras e cereais integrais (aveia, massa, pão, e arroz integral) & $15(32,6)$ & $31(67,4)$ & 0,433 \\
\hline Consumir legumes, frutas e verduras & $31(36,0)$ & $55(64,0)$ & 0,428 \\
\hline Evitar o consumo de açúcar & $29(43,9)$ & $37(56,1)$ & 0,044 \\
\hline Evitar o consumo de doces (guloseimas, biscoitos doces recheados e refrigerantes) & $22(34,9)$ & $41(65,1)$ & 0,001 \\
\hline $\begin{array}{l}\text { Evitar o consumo de frituras, carne vermelha, gorduras visíveis, pele de frango, leite integral, } \\
\text { queijo amarelo }\end{array}$ & $26(33,8)$ & $51(66,2)$ & 0,414 \\
\hline Diminuir o consumo de sal & $31(34,4)$ & $59(65,6)$ & 0,165 \\
\hline Evitar o consumo de embutidos, conservas, enlatados, defumados e salgados de pacote & $18(31,6)$ & $39(68,4)$ & 0,344 \\
\hline Consumir peixes & $21(34,4)$ & $40(65,6)$ & 0,789 \\
\hline
\end{tabular}

* Teste do Qui-quadrado de Pearson.

Ao realizar a associação entre depressão e adesão para cada orientação dos grupos alimentares pode-se observar que a frequência para evitar o consumo de açúcar e de doces foi significativamente menor nos idosos com depressão (Tabela 5).

\section{DISCUSSÃO}

A Organização Mundial da Saúde considera a adesão ineficaz ao tratamento, principalmente no que tange às doenças crônicas, um fenômeno multifatorial com inúmeros determinantes, inter-relacionados $[7-9,19]$. A adesão à dieta, em indivíduos com $\mathrm{SM}$, é fundamental para o manejo da doença, e a interferência de fatores psicológicos, como a depressão, precisa ser considerada. Em outras situações já foi demonstrada associação entre depressão e fatores alimentares [20]. Os dados obtidos com o presente estudo confirmam essa interferência e contribuem para o melhor entendimento desses mecanismos, ao demonstrar especificamente uma associação entre depressão e dificuldade em evitar o consumo de doces e açúcar em idosos com SM.

A frequência da adesão à dieta pelos idosos do presente estudo, avaliada através de um instrumento baseado nas recomendações das diretrizes de $\mathrm{SM}$, foi considerada baixa, pois apenas um terço dos participantes mostraram adesão a todas as recomendações. Estes achados assemelham-se aos resultados de um estudo transversal com adultos e idosos com diabetes mellitus, no qual a adesão foi avaliada por meio da verificação do seguimento às orientações nutricionais determinadas pela Associação Americana de Diabetes (ADA). Aquela investigação evidenciou que apenas metade dos sujeitos aderiu às recomendações da ADA [21].

No entanto, é preciso salientar que a avaliação da adesão é uma tarefa complexa, uma vez que a relação entre as variáveis envolvidas nesse fenômeno ainda não está bem estabelecida [22]. Ressalta-se que não foram encontrados outros estudos que tenham analisado a frequência da adesão à dieta por idosos com SM. Além disso, os diferentes instrumentos utilizados e diferentes pontos de corte para estabelecer a adesão impossibilitam a comparação com os presentes resultados.

A recomendação à qual os idosos apresentaram maior adesão foi a diminuição da ingestão de sal, resultado similar a uma pesquisa sobre a adesão ao tratamento de idosos com hipertensão arterial em uma unidade básica de saúde de João Pessoa, PB, na qual a redução de sal foi também a recomendação com maior taxa de adesão [23]. No estudo de Nolte [24] sobre a frequência da adesão aos 10 passos da alimentação saudável, entre idosos de Dois Irmãos, RS, a autora verificou que a não adição de sal nos alimentos foi o passo que obteve maior seguimento. Uma das explicações seria que a população estudada era composta, em sua maioria, por hipertensos, e pelo fato de a hipertensão arterial poder ser controlada desde que os pacientes se envolvam em ações, principalmente as relacionadas ao controle de dieta hipossódica, com baixo de teor de gorduras e colesterol [25].

Apesar de a maioria dos idosos desta pesquisa estar em condições de sobrepeso, obesidade central, ter história de hipertensão, diabetes mellitus, e dislipidemia, e de afirmar que receberam alguma orientação nutricional, diversas barreiras impediram 
a ação desejada. O sabor desagradável e o preço elevado atribuído aos alimentos tidos como saudáveis [26] foram citados pelos idosos como razões para não adesão à dieta.

Salienta-se que o motivo mais citado pelos idosos do estudo para a não adesão à dieta foi a falta de persistência. Sabe-se que a adesão fica comprometida em situações nas quais os pacientes estão submetidos a tratamentos longos e duradouros [27], como é o caso dos idosos com doenças crônicas, e, principalmente nesses casos, a persistência é fator importante para a manutenção e seguimento do tratamento. A adoção de dietas balanceadas e, principalmente, a redução de sal e açúcar, são consideradas alterações substanciais em práticas alimentares nos idosos, visto que os hábitos alimentares são influenciados pelo contexto familiar, cultural e social, sendo construídos ao longo da vida [28].

Koehnlein et al. [29], ao verificarem a adesão de pacientes de uma clínica particular de nutrição, à reeducação alimentar para a perda de peso, constataram que $40 \%$ dos pacientes abandonaram o tratamento. A mudança drástica dos hábitos alimentares e os resultados lentos foram apontados com umas das dificuldades para seguir a dieta, e as maiores perdas de peso observadas associaram-se aos fatores motivadores.

Em outro estudo de comparação entre dois métodos de intervenção nutricional e o impacto na adesão ao tratamento de pacientes com SM, o autor concluiu que os pacientes que chegaram mais motivados foram aqueles que responderam melhor à redução dos valores do IMC e da circunferência abdominal [30]. A motivação é determinada principalmente pela probabilidade de sucesso, e a frustração pela demora em obter resultados satisfatórios dos seus esforços faz com que os pacientes não se engajem na luta para emagrecer [31].

Em relação à associação entre adesão a todas as orientações dietéticas recebidas (uma a oito) e depressão, não foram observados resultados significativos. Entretanto, ao analisar a associação da depressão com cada item recomendado, os idosos com depressão foram os que aderiram menos às recomendações para evitar o consumo de açúcar e de doces. Este resultado foi semelhante ao encontrado em uma pesquisa na qual a maior ingestão de alimentos doces, de alto valor calórico, foi positivamente associada com sintomas depressivos, em um grupo de 4.655 mulheres com idade entre 40 e 65 anos [32].

Os sintomas depressivos estão associados com mudanças significativas no apetite [18], que podem estar relacionadas a um interesse reduzido no alimento ou, por outro lado, a um aumento do apetite e desejo por doces ou outros alimentos ricos em carboidratos. Os alimentos doces são identificados como os mais atraentes, sendo uma fonte de prazer para os indivíduos, o que pode explicar a grande dificuldade em diminuir ou abolir esses alimentos da dieta $[33,34]$.

Uma das explicações fisiológicas para isso é atribuída ao fato de que determinados hormônios peptídeos agem como neurotransmissores, que aumentam ou diminuem a ingestão alimentar. Concomitantemente, determinados hormônios, como a dopamina, serotonina e ocitocina, são responsáveis pela regulação do humor e afeto. Qualquer desregulação nesses sistemas, ou até mesmo a deficiência ou diminuição dos receptores para esses peptídeos, pode influenciar o humor e o comportamento. No enfrentamento de situações estressoras ou de desgaste emocional, indivíduos que sofrem algum transtorno de humor podem aumentar o consumo de alimentos altamente palatáveis, como doces e carboidratos, de forma a compensar as deficiências provocadas pela desregulação desses hormônios [34-37].

Para uma melhor adesão à dieta, é muito importante que o paciente tenha conhecimento da composição dos alimentos, como atuam no organismo, e quais as proporções ideais de consumo. Estar a par dessas informações influencia positivamente no seguimento das prescrições, e o desconhecimento do assunto, por sua vez, pode dificultar a adesão ao tratamento. Em uma metanálise, que teve como propósito investigar novas estratégias para que indivíduos portadores de SM modifiquem o seu estilo de vida, os autores observaram que o aconselhamento pelos profissionais de saúde pode aumentar significativamente a motivação em adquirir hábitos saudáveis de vida, incluindo a dieta [38]. Nesse sentido, torna-se importante, também, conhecer os sintomas de estresse psicológico e de depressão, visto que esses aspectos podem influenciar significativamente a adesão para mudar comportamentos [39].

Destaca-se a importância do trabalho da equipe multiprofissional de saúde na atenção básica, na realização de atividades de educação em saúde com os idosos, envolvendo aspectos ligados ao reconhecimento e à aceitação de suas condições de saúde, habilitandoos à identificação de fatores de risco no estilo de vida, e à importância do autocuidado, principalmente naqueles acometidos por algum transtorno depressivo. A avaliação da adesão ao tratamento não é uma tarefa considerada fácil, visto que está relacionada aos fatores comportamentais, não significando apenas observar o cumprimento das orientações estabelecidas pelos profissionais, mas sim conhecer os motivos, fatores e contextos sociais e culturais, bem como as doenças associadas. 
Como principal limitação deste estudo, ressaltase seu caráter transversal. Outra limitação refere-se ao caráter dicotômico do instrumento utilizado para avaliação da adesão à dieta.

Conclui-se que nos idosos diagnosticados com SM, atendidos na atenção básica à saúde, apenas um terço era aderente à dieta, ou seja, seguia todas as recomendações dietéticas recebidas de um profissional da saúde. Nos indivíduos com diagnóstico de depressão, a adesão às orientações de evitar o consumo de doces e de açúcar foi menor.
Espera-se que estes resultados forneçam subsídios para que as equipes da atenção básica possam estabelecer medidas estratégicas para melhora na adesão às recomendações dietéticas por idosos com SM, com o objetivo de controlar a doença já instalada e diminuir os riscos de complicações. Como perspectiva, pretende-se conduzir estudos com métodos mistos (qualitativos-quantitativos), além de elaborar um instrumento com escala de intervalo ou escala de Lickert, para aprofundar a compreensão sobre o comportamento de idosos em relação à adesão à dieta.

\section{NOTAS}

Agradecimentos

Os autores agradecem a todos os pesquisadores do EMI-SUS.

Apoio financeiro

Este estudo recebeu auxílio financeiro da Fundação de Amparo à Pesquisa do Estado do Rio Grande do Sul (FAPERGS). C. B. J. recebeu bolsa de estudo da PUCRS e V. E. C. da Coordenação de Aperfeiçoamento de Pessoal de Nível Superior (CAPES).

Declaração de conflitos de interesse

Os autores declaram não haver conflitos de interesse relevantes ao conteúdo deste estudo.

\section{REFERÊNCIAS}

1. World Health Organization. Global status report on noncommunicable diseases. [Internet]. Geneva: WHO; 2014 [cited 2016 Jan 18]. Available from: http://apps.who.int/iris/bitstream/10665/148114/1/9789241564854_eng.pdf

2. Ferro MA. Major depressive disorder, suicidal behaviour, bipolar disorder, and generalized anxiety disorder among emerging adults with and without chronic health conditions. Epidemiol Psychiatr Sci. 2015 Sept 8:1-13. http://dx.doi.org/10.1017/ S2045796015000700

3. Rodrigues GHP, Gebara OCE, Gerbi CCS, Pierri P, Wajngarten M. Depressão como determinante clínico de dependência e baixa qualidade de vida em idosos cardiopatas. Arq Bras Cardiol. 2015;104(6):443-9.

4. I Diretriz Brasileira de Diagnóstico e Tratamento da Síndrome Metabólica. Arq Bras Cardiol. 2005;84(Supl 1):1-28. http://dx.doi.org/10.1590/S0066-782X2005000100001

5. Grundy SM. Metabolic syndrome update. Trends Cardiovasc Med. [In Press] Dallas; 2015 [cited 2016 Jan 18]. Available from: http://www.sciencedirect.com/science/article/pii/S1050173815002492

6. Soares TS, Haas C, Gustavo AS, Macagnan FE, Bodanese LC, Feoli AMP. Hábitos alimentares, atividade física e Escore de Risco Global de Framingham na síndrome metabólica. Arq Bras Cardiol. 2014;102(4):374-82. http://dx.doi.org/10.5935/ abc. 20140029

7. World Health Organization. Defining Adhrence. In: World Health Organization. Adhrence to long-term therapies: evidence for action. Geneva: WHO; 2003. p. 192-4.

8. Organización Mundial de La Salud. Delimitación del problema In: Organización Panamericana de La Salud. Adherencia a lós tratamentos a largo plazo: pruebas para la acción. Washington: OMS; 2004. p. 3-47.

9. Boas LCGV, Foss MC, Foss-Freitas MCF, Torres HC, Monteiro LZ, Pace AE. Adesão à dieta e ao exercício físico das pessoas com diabetes mellitus. Texto Contexto Enferm. 2011;20(2):272-9. http://dx.doi.org/10.1590/S0104-07072011000200008

10. Gillen MM, Markey CN, Markey PM. An examination of dieting behaviors among adults: links with depression. Eat Behav. 2012 Apr;13(2):88-93. http://dx.doi.org/10.1016/j.eatbeh.2011.11.014

11. Moraes AL, Almeida EC, Souza LB. Percepções de obesos deprimidos sobre os fatores envolvidos na manutenção da sua obesidade: investigação numa unidade do Programa Saúde da Família no município do Rio de Janeiro. Physis. 2013;23(2): 553-72. http://dx.doi.org/10.1590/S0103-73312013000200012

12. Gomes I, Nogueira EL, Engroff P, Ely LS, Schwanke CHA, De Carli GA. The multidimensional study of the elderly in the family health strategy in Porto Alegre, Brazil (EMI-SUS). Pan Am J Aging Res. 2013;1(1):20-4. 
13. Expert Panel on Detection, Evaluation and Treatment of High Blood Cholesterol in Adults. Executive Summary of the Third Report of the National Cholesterol Education Program (NCEP) Expert Panel on Detection, Evaluation, and Treatment of High Blood Cholesterol in Adults (Adult Treatment Panel III). JAMA. 2001 May 16;285(19):2486-97. http://dx.doi.org/10.1001/ jama.285.19.2486

14. Jacondino CB. Adesão ao tratamento medicamentoso e não medicamentoso em idosos portadores de síndrome metabólica acompanhados na Estratégia Saúde da Família [dissertação]. [Porto Alegre]: Pontifícia Universidade Católica do Rio Grande do Sul; 2013. 139 p.

15. Lipschitz DA. Screening for nutritional status in the elderly. Prim Care. 1994 Mar;21(1):55-67.

16. Sociedade Brasileira de Patologia Clínica. Recomendações da Sociedade Brasileira de Patologia Clínica. Medicina laboratorial para coleta de sangue venoso. $2^{\mathrm{a}}$ ed. Barueri: Manole; 2009.

17. Sociedade Brasileira de Cardiologia. VI Diretrizes Brasileiras de Hipertensão. Arq Bras Cardiol. 2010;95(supl. 1):1-51.

18. American Psychiatric Association Committee on Nomenclature and Statistics Diagnostics. Diagnostic and Statistical Manual of Mental Disorders (DSM-IV). 4th ed. Washington DC; 1994.

19. Rodriguez GMJ, Castillo AF. Enfermeras y adherencia farmacológica: discurso y conducta. Index Enferm. 2011;20(3):160-4. http://dx.doi.org/10.4321/S1132-12962011000200005

20. Fuemmeler BF, Agurs-Collins T, McClernon FJ, Kollins SH, Garrett ME, Ashley-Koch AE. Interactions between genotype and depressive symptoms on obesity. Behav Genet. 2009 May;39(3):296-305. http://dx.doi.org/10.1007/s10519-0099266-Z

21. Mu-oz-Pareja M, León-Mu-oz LM, Guallar-Castillón P, Graciani A, López-García E, Banegas JR, Rodríguez-Artalejo F. The diet of diabetic patients in Spain in 2008-2010: accordance with the main dietary recommendations - a cross-sectional study. PLoS One. 2012;7(6):e39454. http://dx.doi.org/10.1371/journal.pone.0039454

22. Zanetti ML, Arrelias CCA, Franco RC, Santos MA, Rodrigues FFL, Faria HTG. Adesão às recomendações nutricionais e variáveis sócio demográficas em pacientes com diabetes mellitus. Rev Esc Enferm USP. 2015;49(4):619-25. http://dx.doi. org/10.1590/S0080-623420150000400012

23. Dourado CS, Costa KNF, Oliveira JS, Leadebal ODC, Silva LS. Adesão ao tratamento de idosos com hipertensão arterial em uma unidade básica de saúde de João Pessoa PB. Acta Sci Health. 2011;33(1):9-17.

24. Nolte AO. Associação entre hábitos saudáveis e alimentares, estado nutricional e percepção de saúde dos idosos [dissertação]. [Porto Alegre]: Pontifícia Universidade Católica do Rio Grande do Sul; 2011.98p.

25. Tavares NUL, Bertoldi AD, Thume E, Facchini, LA, Franca GVA, Mengue, SS. Fatores associados a baixa adesão ao tratamento medicamentoso em idosos. Rev Saúde Publ. 2013;47(6):1092-101. http://dx.doi.org/10.1590/S0034-89102013000901092

26. Leão JM, Lisboa LCV, Pereira MAL, Freitas L, Lacerda KC, Elias MAR. Estágios motivacionais para mudança de comportamento em indivíduos que iniciam tratamento para perda de peso. J Bras Psiquiatr. 2015;64(2):107-14. http://dx.doi.org/10.1590/00472085000000065

27. Arruda DCJ, Eto FN, Velten APC, Morelato RL, Oliveira ERA. Fatores associados a não adesão medicamentosa entre idosos de um ambulatório filantrópico do Espírito Santo. Rev Bras Geriat Gerontol. 2015;18(2):327-37. http://dx.doi.org/10.1590/18099823.2015.14074

28. Pontieri FM, Bachion MM. Crenças de pacientes diabéticos acerca da terapia nutricional e sua influência na adesão. Ciên Saúde Colet. 2010;15(1):151-60. http://dx.doi.org/10.1590/S1413-81232010000100021

29. Koehnlein EA, Salado GA, Yamado, NA. Adesão à reeducação alimentar para a perda de peso: determinantes, resultados e a percepção do paciente. Rev Bras Nutr Clin. 2008;23(1):56-65.

30. Busnello FM, Bodanese LC, Pellanda LC, Santos ZEA. Intervenção nutricional e o impacto na adesão ao tratamento em pacientes com síndrome metabólica. Arq Bras Cardiol. 2011;97(3):217-24. http://dx.doi.org/10.1590/S0066-782X2011005000077

31. Vieira CM, Cordeiro SN; Magdaleno Júnior R, Turato ER. Significados da dieta e mudanças de hábitos para portadores de doenças metabólicas crônicas: uma revisão. Ciên Saúde Colet. 2011,16(7):3161-8. http://dx.doi.org/10.1590/S141381232011000800016

32. Jeffery RW, Linde JA, Simon GE, Ludman EJ, Rohde P, Ichikawa LE, Finch EA. Reported food choices in older women in relation to body mass index and depressive symptoms. Appetite. 2009 Feb;52(1):238-40. http://dx.doi.org/10.1016/j. appet.2008.08.008

33. Konttinen H, Männistö S, Sarlio-Lähteenkorva S, Silventoinen K, Haukkala A. Emotional eating, depressive symptoms and self-reported food consumption. A population-based study. Appetite. 2010 Jun;54(3):473-9. http://dx.doi.org/10.1016/j. appet.2010.01.014

34. Rahe C, Baune BT, Unrath M, Arolt V, Wellmann J, Wersching H, Berger K. Associations between depression subtypes, depression severity and diet quality: cross sectional findings from the BiDirect Study. BMC Psychiatry. 2015 Mar 4;15:38. http://dx.doi.org/10.1186/s12888-015-0426-9

35. Feijó FM, Bertolucci MC, Reis C. Serotonina e controle hipotalâmico da fome: uma revisão. Rev Assoc Med Bras. 2011;57(1): 74-7. http://dx.doi.org/10.1590/S0104-42302011000100020 
36. Jacondino CB, Gottlieb MGV. Oxytocin and chronic noncommunicable diseases: on overview of oxytocin receptor gene (OXTR) polymorphisms and the thrifty phenotype hypothesis. In: Anderson E, editors. Advances in oxytocin research: pharmacology - research, safety, testing and regulation. Nova York: Nova Science Publishers; 2015. p. 1-34.

37. Agurs-Collins T, Fuemmeler BF. Dopamine polymorphisms and depressive symptoms predict foods intake: results from a nationally representative sample. Appetite. 2011 Oct;57(2):339-48. http://dx.doi.org/10.1016/j.appet.2011.05.325

38. Bassi N, Karagodin I, Wang S, Vassallo P, Priyanath A, Massaro E, Stone NJ. Lifestyle modification for metabolic syndrome: a systematic review. Am J Med. 2014 Dec;127(12):1242.e1-10. http://dx.doi.org/10.1016/j.amjmed.2014.06.035

39. Durrani S, Irvine J, Nolan RP. Psychosocial determinants of health behaviour change in an counseling intervention for hypertension. Int J Hypertens. 2012;2012:191789. C 